\title{
DESIGN AND CONTROL OF CAPSTAN-PULLEY DRAW SYSTEM FOR OPTICAL FIBER MANUFACTURING
}

\author{
Hodge E. Jenkins \\ Dept. of Mechanical and Industrial Engineering \\ Mercer University \\ 1400 Coleman Ave. \\ Macon, GA 31207 USA \\ jenkins_he@mercer.edu
}

\author{
Mark L. Nagurka \\ Dept. of Mechanical Engineering \\ Marquette University \\ 1515 W. Wisconsin Ave. \\ Milwaukee, WI 53233 USA \\ mark.nagurka@marquette.edu
}

\begin{abstract}
Optical fiber for data communication is manufactured by the draw process, which involves heating and pulling high purity glass cylinders to diameters of $125 \mu \mathrm{m}$. The diameter of the glass fiber and its light-guide core must remain constant to create a product capable of transmitting high-bandwidth optical data. The draw capstan design has a significant impact on the optical fiber quality. As the draw speed is used to control the fiber diameter, the ability of the draw capstan to follow velocity commands directly affects the resulting fiber diameter.

To improve the control of the optical fiber diameter, the design of the overall system was revisited. A lumped-parameter model of the capstan drive was developed. It accounts for disturbances in the draw process that arise from sources such as the variation in the diameter of the input glass cylinder and the draw tension control, affecting the glass temperature and viscosity. The selection of the motor and the design of the speed controller in the optical fiber draw capstan pulley system were studied. Simulation studies over a range of parameters demonstrate that speed regulation, necessary to manufacture optical fiber within allowable diameter tolerances, can be achieved in the presence of estimated process disturbances without motor current saturation. The model predictions suggest that an effective draw capstan system can be synthesized and controlled.

This case study has broad applications for the design of practical engineering systems that include control, electrical, and mechanical subsystems, typical in modern manufacturing. The paper highlights the use of mechanical and electrical modeling, system identification, and control design as necessary parts of product and process improvement.
\end{abstract}

\section{INTRODUCTION}

The design and control of an optical fiber draw capstan system highlights modeling and design challenges often faced in solving mechatronic engineering problems. The design problem is multi-faceted, and entails system identification, system modeling, practical mechanical design, motor selection, and closed-loop control system design. Due to inherent tradeoffs, typical in engineering problems, the design and performance of the capstan system are linked. The operation of the capstan system can be improved through an integrated, systems-level understanding predicated on engineering fundamentals and tools from multiple technical disciplines.

An objective of this case study is to help reduce boundaries - artificial and real - dividing engineering disciplines by encouraging a blended problem-solving approach that draws upon several areas of technical knowledge and competence. The design problem here is solved with tools from mechanical, electrical, and control engineering.

The name "mechatronic design" is often ascribed to design challenges in industrial applications that encompass multiple engineering disciplines and backgrounds. Real-life examples of mechatronic designs include auto-focus cameras, CD players, and robotic vacuum cleaners. In general, machines and processes that rely on sensors, actuators, mechanisms, controllers and micro-processors of various types, sizes, and attributes are described as mechatronic systems. Large-scale systems, such as industrial plants and vehicles, with many control loops, subsystems, and inter-connections under computer control are at one end of the mechatronic spectrum and relatively simpler devices such as magnetic bearings are at the other extreme [1-3]. 


\section{DRAW PROCESS BACKGROUND}

Optical fiber is a light guide providing high-speed data transmission at the terabit $\left(10^{12}\right)$ per second level [4]. Moving light through the glass fiber core relies upon the principle of total internal reflection, where the inside glass core has a higher refractive index $\left(\mathrm{n}_{1}\right)$ than the cladding glass $\left(\mathrm{n}_{2}\right)$ around the core, as shown in Fig. 1.

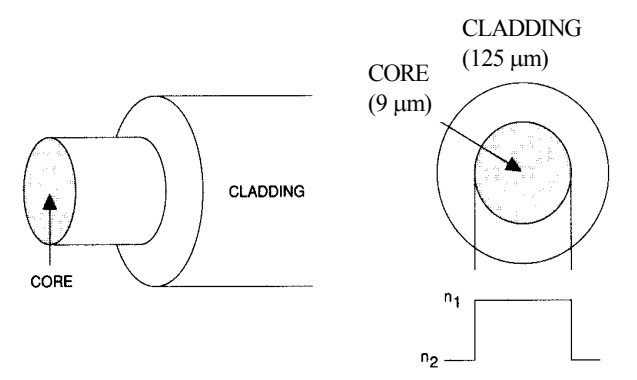

Figure 1. Typical glass components of optical fiber

Optical fiber is manufactured by the draw process. This process is the only cost-effective means of creating optical fiber from ultra-high purity glass cylinders with the required refractive index profile [4-6]. An important manufacturing quality concern is maintaining a constant core diameter for efficient light transmission. Core diameter variations lead to dispersion of light and loss of signal strength.

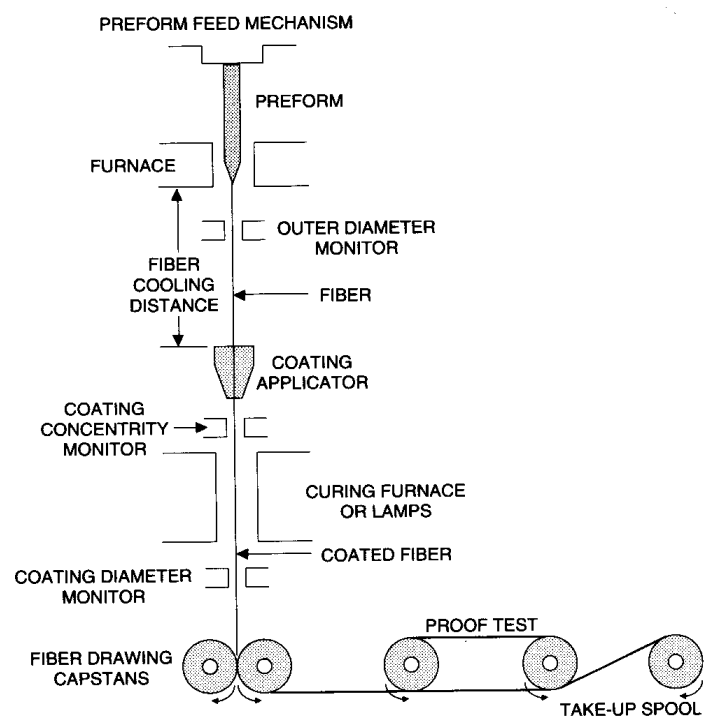

Figure 2. Draw manufacturing process for optical fiber

Figure 2 illustrates the draw manufacturing process for optical fiber. A high purity glass cylinder with a prescribed optical index profile, known as a preform, is fed into a specially designed furnace and heated to the point where the glass flows under low tension. The draw capstan pulls the fiber from the bottom of the glass preform in the furnace; the glass preform feed mechanism above the furnace maintains material flow equilibrium through the furnace. The fiber is then cooled, coated with protective polymers, cured under ultraviolet lights, and wound onto spools.

The capstan controls the diameter of the fiber by adjusting the speed at which the fiber is drawn. Measurement of the input glass diameter occurs just below the furnace. (Cooling occurs rapidly in the thin fiber, so thermal expansion of the fiber relative to room temperature is inconsequential.) The capstan speed, i.e., the draw speed, controls the fiber diameter: the speed is increased when the fiber is too thick and decreased when the fiber is too thin. (The temperature of the furnace affects the pulling tension on the fiber by the capstan. The furnace temperature set point is adjusted based on the measured fiber tension. This tension is important in the draw process, and a full discussion is beyond the scope of this paper.) .

The basic design of the fiber draw capstan is a flexible belt partially wound over a flat pulley that moves/pulls a continuous optical fiber all the way from the heated preform, as shown in Fig. 3. Tests have validated a relationship between the fiber diameter and the line speed about an operating point. The basis of the relationship is constant volumetric flow rate of glass.

Variations in fiber diameter arise quite often from process disturbances, such as non-uniformity of the preform diameter, mismatches of the preform feed volumetric glass input relative to that of the drawn fiber, and drifts in the furnace/glass temperature. Thus, in addition to the mechanical design and motor selection, a speed controller is needed in the capstan design to prevent impermissible variations in the fiber diameter from process disturbances.

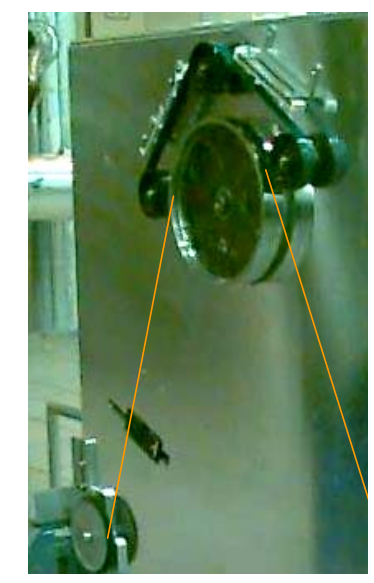

Figure 3. Optical fiber drawn by capstan

\section{DESIGN SPECIFICATIONS AND PARAMETERS}

The target dimensions of the optical fiber glass are an outside diameter of $125 \mu \mathrm{m}$ and an inside core diameter of $9 \mu \mathrm{m}$. Product specifications call for outside diameter tolerances of \pm 1 $\mu \mathrm{m}$. To achieve and improve upon this product specification, the permissible diameter error is set to a lower design deviation target of $\pm 0.1 \mu \mathrm{m}(\sim 0.1 \%)$. The capstan mechanical, electrical, and control designs synergistically impact the ability to achieve this fiber diameter tolerance. 
With the permissible diameter deviation specified, the next step is to understand how the selected parameters of the capstan design affect the resulting product. While many decisions must be made in any design, there are usually a limited number of critical parameters that have significant influence on operational performance. For the capstan design problem, these parameters include:

- The diameter and tolerances of the capstan pulley

- Inertia limits

- Belts, contact length, and bearings

- Torques and speeds of the motors

- Control gains

- Maximum current and power limits of the amplifier

- Sensor noise

In this mechatronic system, both the mechanical design and the control design influence the overall system performance. The primary mechanical design decision is the diameter of the capstan pulley. The required line speed and machining tolerances are the basis of the design. If no disturbances are present, the established design parameters determine the maximum possible variations in diameter.

A typical capstan drive is shown in Fig. 4. Belt tension, damping, and bearing loads are present. The tension in the belt is significantly higher than the tension to pull the fiber. Dynamic effects of the capstan belt and bearings are treated as viscous damping and additional inertia.

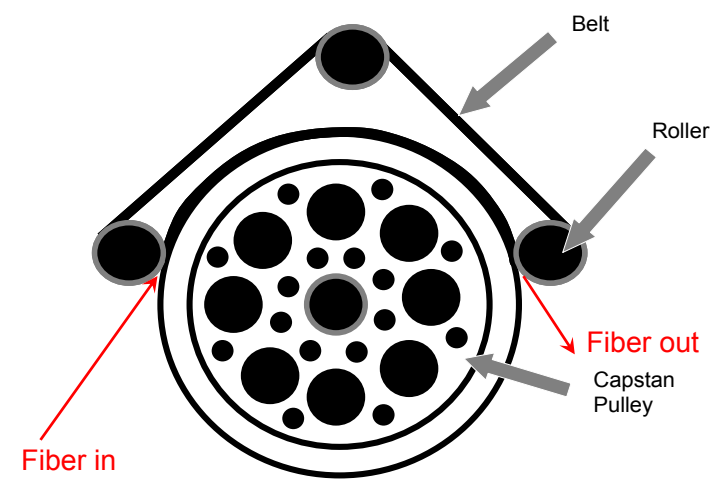

Figure 4. Capstan pulley with belt with three rollers

Although the belts are not the focus of this case study, they are a key component of the design. The belt material, contact length, and belt tension must be selected appropriately to achieve non-slip belt movement with respect to the capstan pulley. The belt/capstan friction and prevention of fiber coating damage are among other central considerations.

Capstan drives with smaller diameters are preferred. In addition to a lower mass moment of inertia and faster dynamic response with less control effort, they have less material and lower machining costs. The diameter of the capstan pulley is limited on the lower end by the mechanical strength of the fiber under tension and bending. Experiments have shown that the smallest allowable diameter is $75 \mathrm{~mm}$. The diameter selection must also consider machining tolerances and maximum motor speeds. Half of the design error $(0.05 \%)$ is budgeted to the mechanical tolerances and half is allocated to the control design. Available machining tolerances of $\pm 0.05 \mathrm{~mm}$ for the diameter indicate that a diameter of $100 \mathrm{~mm}$ or larger must be used to meet the maximum design error, as seen in Fig. 5. The mass moment of inertia of the capstan pulley is a quadratic function of diameter, as seen in Fig. 6. The lower the pulley inertia, the smaller the required control effort and power. As such, smaller diameter pulleys are more desirable given the control and inertia effects.

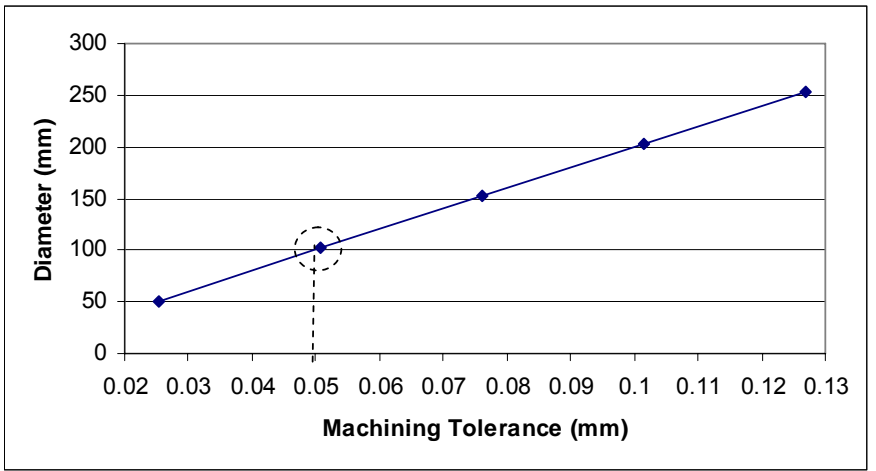

Figure 5. Pulley diameter vs. machining tolerance for $0.05 \%$ radius variation

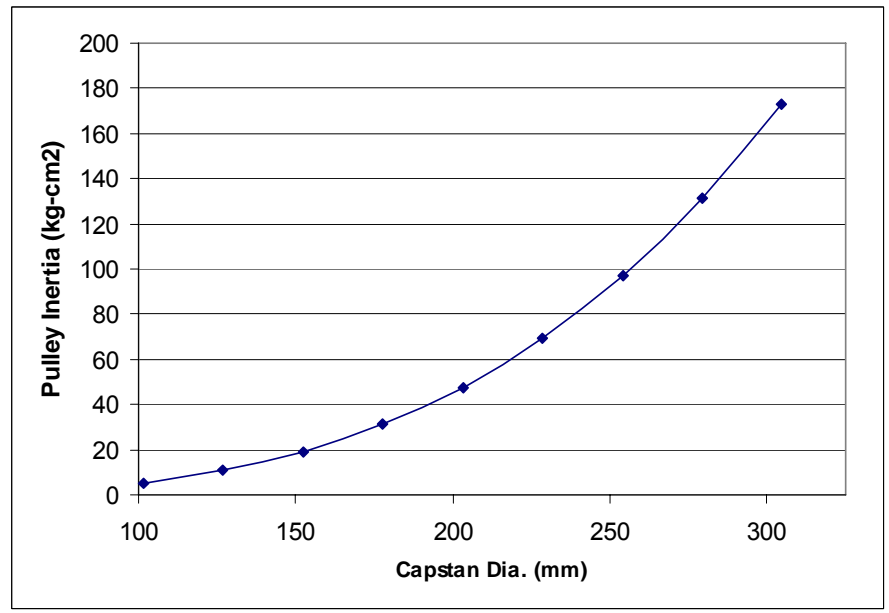

Figure 6. Capstan pulley inertia vs. diameter

Another important consideration in the capstan design is the maximum speed of the motor. To allow for diameter control at a normal draw line speed of $50 \mathrm{~m} / \mathrm{s}$, the maximum line speed must be higher. Figure 7 depicts the relationship between motor RPM and pulley diameter for a maximum operational draw line speed of $60 \mathrm{~m} / \mathrm{s}$. Based on speed requirements, two potential diameters are possible. For a motor with a limit of 4,000 RPM a pulley diameter of approximately $300 \mathrm{~mm}$ (or larger) is required. A pulley diameter of about $200 \mathrm{~mm}$ is the minimum diameter necessary for a 6,000 RPM limit. With previously 
established design preferences for smaller diameter pulleys, the $200 \mathrm{~mm}$ diameter pulley is the logical choice if a motor with a maximum speed of 6,000 RPM or greater is available and meets the electrical requirements.

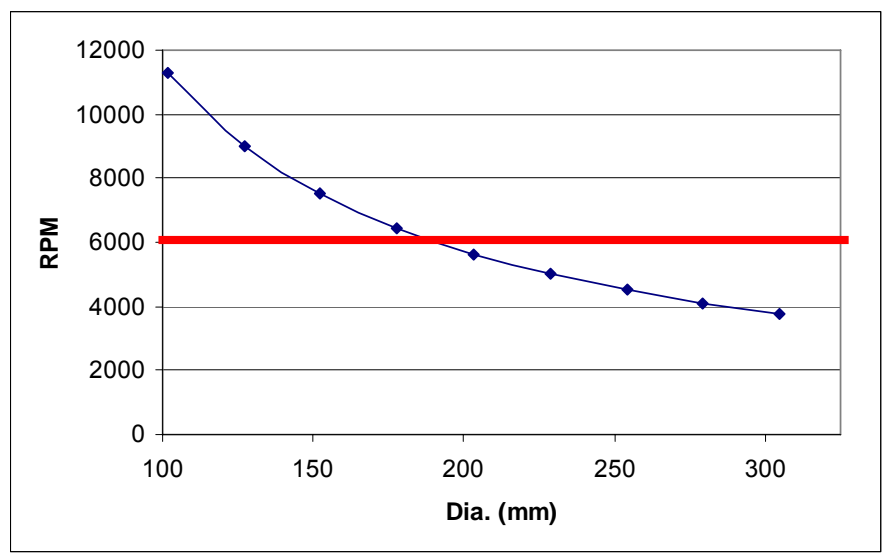

Figure 7. Motor RPM vs. pulley diameter for $60 \mathrm{~m} / \mathrm{s}$ line speed

\section{MOTOR SELECTION}

A motor can be selected for each of the two possible diameters (200 $\mathrm{mm}$ and $300 \mathrm{~mm}$ ) of the capstan pulley. The choice of motor depends on several factors including maximum speed, torque, inertia, current, and power limitations. The motor must handle the controller amplifier power limits of $800 \mathrm{~W}$ per axis continuous and $1600 \mathrm{~W}$ peak. The rated motor current must be greater than the maximum amplifier current of $10 \mathrm{~A}$ to prevent overheating. Specifications for motors compatible with the specified controller and amplifier are presented in Table 1. The most distinguishing parameters for the listed motors are maximum speed and torque constants. Current and power limits are generally affected by accelerations of line speed. Given the amplifier limits, most of the motors will not approach their current and power limitations during operation at line speed.

From Table 1, motors 3, 5, or 7 achieve the speed $(6,000$ RPM), power, and current requirements for a $200 \mathrm{~mm}$ diameter pulley. Motor 3 is selected because of its relatively lower rotor inertia and approximately equivalent torque constant. For the $300 \mathrm{~mm}$ diameter pulley design, a motor rated at 4,000 RPM or higher is required. Motors 4 or 10 would be acceptable. While motor 4 is slightly below the maximum current limit specification (9.6 A), it has a $20 \%$ higher torque constant in comparison to motor 10 . Thus, there are two candidate capstan designs: a $200 \mathrm{~mm}$ diameter pulley with motor 3 or a $300 \mathrm{~mm}$ diameter pulley with motor 4 . The capstan design with the smaller pulley (200 $\mathrm{mm}$ diameter) is preferred.
Table 1. Specifications for Available Motors [7]

\begin{tabular}{|l|c|c|c|c|c|c|c|}
\hline Model & Motor & $\begin{array}{c}\text { Power } \\
\text { (kW) }\end{array}$ & $\begin{array}{c}\text { Rated } \\
\text { Torque } \\
\mathbf{( N - m )}\end{array}$ & $\begin{array}{c}\text { Rated } \\
\text { Speed } \\
\text { (RPM) }\end{array}$ & $\begin{array}{c}\text { Torque } \\
\text { Constant } \\
\text { (N-m/ A) }\end{array}$ & $\begin{array}{c}\text { Rated } \\
\text { Current } \\
\text { (Amps) }\end{array}$ & $\begin{array}{c}\text { Rotor } \\
\text { Inertia (kg } \\
\mathbf{~ ( m 2 ) ~}\end{array}$ \\
\hline ASM121A-5 & 1 & 1.75 & 2.2 & 4,000 & 0.5 & 5 & 5.7 \\
\hline ASM121A-22 & 2 & 2.555 & 2.2 & 6,000 & 0.3 & 7.3 & 5.7 \\
\hline ASM121B-11 & 3 & 4.97 & 4.4 & 6,000 & 0.31 & 14.2 & 9.9 \\
\hline ASM121B-16 & 4 & 3.36 & 4.4 & 4,000 & 0.46 & 9.6 & 9.9 \\
\hline ASM121C-7 & 5 & 7.7 & 6.6 & 6,000 & 0.3 & 22 & 12.9 \\
\hline ASM121C-14 & 6 & 3.85 & 6.6 & 3,000 & 0.6 & 11 & 12.9 \\
\hline ASM143A-08 & 7 & 11.2 & 9.2 & 6,000 & 0.29 & 32 & 22 \\
\hline ASM143A-14 & 8 & 6.23 & 9.2 & 3,000 & 0.52 & 17.8 & 22 \\
\hline ASM143A-16 & 9 & 5.25 & 9.2 & 3,000 & 0.62 & 15 & 22 \\
\hline ASM143A-40 & 10 & 8.155 & 9.2 & 4,000 & 0.39 & 23.3 & 22 \\
\hline
\end{tabular}

\section{DERIVATION OF EQUATIONS OF MOTION}

The dynamic equations of motion for the capstan pulley system are developed for the model shown in Fig. 8.

$$
\sum T(t)=J_{\text {total }} \dot{\omega}
$$

or

$$
J_{\text {total }} \dot{\omega}=-B_{\text {total }} \omega+T_{\text {motor }}
$$

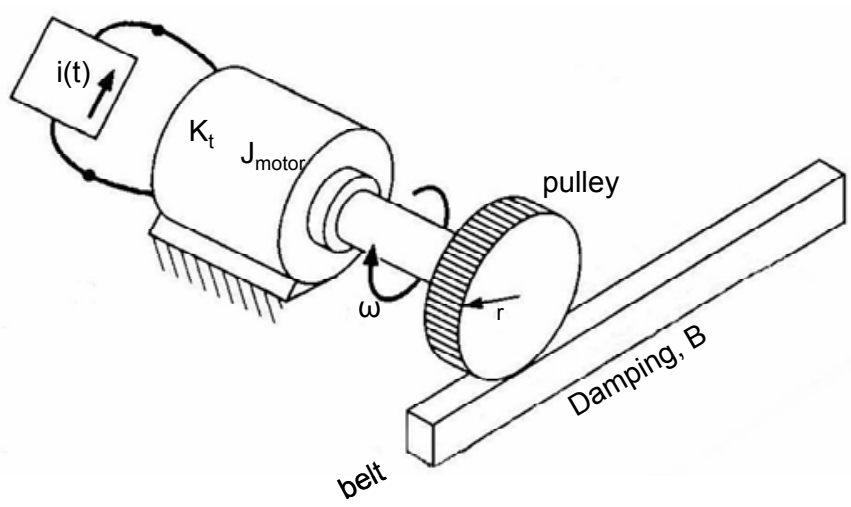

Figure 8. Idealized optical fiber draw capstan pulley (adapted from [9])

The motor torque is a function of the applied current.

$$
T_{\text {motor }}=K_{t} i(t)
$$

Substituting Eq. (3) into Eq. (1) yields Eq. (4).

$$
J_{\text {total }} \dot{\omega}=-B_{\text {total }} \omega+K_{t} i(t)
$$

The effective mass moment of inertia about the capstan rotation center is the sum of the inertia of the motor rotor, pulley, belt and rollers.

$$
J_{\text {total }}=J_{\text {motor }}+J_{\text {pulley }}+J_{\text {belt }}+J_{\text {rollers }}
$$


The capstan pulley can be modeled as a rim and a flange, i.e.,

$$
J_{\text {pulley }} \cong M_{\text {rim }} r^{2}+\frac{1}{2} M_{\text {flange }}(r-2 \tau)^{2}
$$

The mass moment of inertia for the belt and three rollers is expressed as

$$
J_{\text {belt }}+J_{\text {rollers }}=M_{\text {belt }} r^{2}+3\left(\frac{1}{2} M_{\text {roller }} r_{\text {roller }}^{2}\right) .
$$

The effective damping of the system is the sum of the damping from the bearings and belt.

$$
B_{\text {total }}=B_{\text {belt }}+B_{\text {bearings }}
$$

The damping is difficult to calculate but can be estimated by input-output relationships. Reformulating Eq. (4)

$$
\dot{\omega}+\frac{B_{\text {total }}}{J_{\text {total }}} \omega=\frac{K_{t}}{J_{\text {total }}} i(t)
$$

Taking the Laplace transform of Eq. (9) with no initial conditions

$$
s \Omega(s)+\frac{B_{\text {total }}}{J_{\text {total }}} \Omega(s)=\frac{K_{t}}{J_{\text {total }}} I(s)
$$

From Eq. (10), the transfer function between speed and current may be derived

$$
G_{M}(s)=\frac{\Omega(s)}{I(s)}=\frac{\frac{K_{t}}{J_{\text {total }}}}{s+\frac{B_{\text {total }}}{J_{\text {total }}}}
$$

Using Eq. (11), the total damping (Eq. (8)) may be estimated from a current step input and velocity data via system identification tools [8]. The root (or pole) of the first order system of the Eq. (11) is real and negative. Because damping values are typically small, the response of the motor is generally relatively slow and control is necessary for effective disturbance rejection.

\section{CONTROL DESIGN AND PARAMETERS}

Figure 9 represents the block diagram for the diameter and speed control of the draw capstan. The approach is a cascaded controller of an outer fiber diameter control loop with an inner capstan speed control loop. The diameter error is used to change the capstan speed set point, based on constant volumetric flow. The volumetric flow rate out of the furnace is the square of the fiber diameter multiplied by the capstan pull speed and $\pi / 4$. A positive diameter error requires a positive speed increase to lower the diameter. The motor speed change from the diameter variation is relatively small. The speed change is added to the original speed set point. Thus, the fiber diameter control is fine tuning the velocity set point.

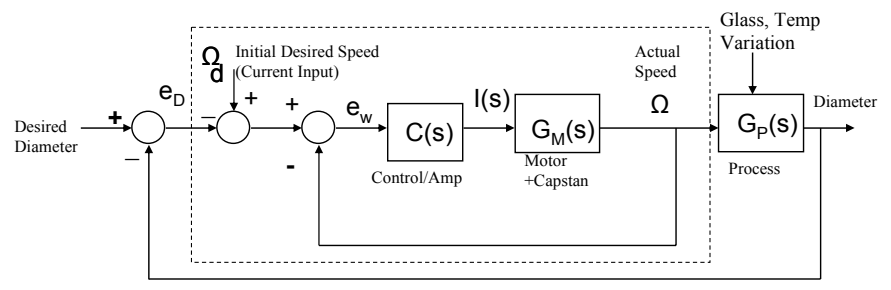

Figure 9. Capstan speed is controlled within a cascaded control loop for fiber diameter

The open-loop capstan speed transfer function, $G_{M}(s)$, is a first order system and yields an exponential function of time for a step change in current.

$$
\omega(t)=\Delta I\left(\frac{K_{t}}{J_{\text {total }}}\right) \cdot\left[1-e^{-\left(B_{\text {total }} / J_{\text {total }}\right) \cdot t}\right]
$$

Although proportional control alone with a high gain can reduce the error, the steady-state error can be eliminated by using a compensator with proportional plus integral (PI) control.

$$
C(s)=K_{p}\left(s+\frac{K_{i}}{K_{p}}\right) \frac{1}{s}
$$

In the creation of fiber the basic premise of capstan operation and speed control is constant volumetric glass flow out of the furnace, $Q$.

$$
Q=\frac{1}{4} \pi r \omega_{d} d_{\text {act }}^{2}=\frac{1}{4} \pi r \omega_{\text {nom }} d_{\text {nom }}^{2}
$$

The control logic is as follows: increase the capstan speed set point if the fiber diameter is too thick or decrease it if it is too small.

$$
\omega_{d}=\omega_{\text {nom }}\left(\frac{d_{\text {nom }}}{d_{\text {act }}}\right)^{2}
$$

Typical variations in the diameter of the glass preform can be approximated as a $2 \%$ change over $10 \mathrm{~mm}$ of the preform length. The shape assumed for the preform diameter deviation is a linear ramp. The ratio of preform diameter to fiber diameter is approximately $700: 1$. Squaring this gives a volumetric ratio of 490,000:1. Thus, the time for drawing $10 \mathrm{~mm}$ of preform (or $4900 \mathrm{~m}$ of fiber) is approximately $100 \mathrm{~s}$ (at a fiber line speed of $50 \mathrm{~m} / \mathrm{s}$ ). Squaring the diameter error yields a $4 \%$ volumetric variation over $10 \mathrm{~mm}$ of preform length. This volume error is accommodated in the control design by changing the draw speed set point. Assuming a linear ramp function for the 
preform diameter (input), the line speed of the fiber would require a speed change ramp of $0.04 \%$ per second to keep the fiber diameter constant. In addition, this corresponds to $1.25 \mathrm{~s}$ for an uncontrolled fiber diameter to deviate to half of the tolerance budget $(0.05 \%$ deviation). Thus, it is desirable to keep the settling time under one second for the inner speed loop to reduce the dynamic effects on the outer control loop.

\section{CONTROLLER ELECTRICAL PARAMETERS}

To ensure the capstan design performs as expected, it is necessary to identify the controller electrical specifications and verify that they are not exceeded. Amplifier power is limited to $800 \mathrm{~W}$ per axis continuous and $1600 \mathrm{~W}$ peak. Current is limited to $10 \mathrm{~A}$ per axis. The controller maximum update rate is 20 $\mathrm{kHz}$. With these performance specifications, summarized in Table 2, the capstan and controller design can be simulated. The selected $200 \mathrm{~mm}$ capstan pulley design with motor 3 can then be assessed.

Table 2. Summary of Controller/Amplifier Specifications for Capstan Axis

\begin{tabular}{|l|l|}
\hline Maximum Power & $1600 \mathrm{~W}$ peak; $800 \mathrm{~W}$ continuous \\
\hline Maximum Current & $10 \mathrm{~A}$ \\
\hline Settling time & $1.0 \mathrm{~s}$ or less \\
\hline Sample rate & 1 to $20 \mathrm{kHz}$ \\
\hline
\end{tabular}

The open loop transfer function for the capstan pulley, motor, and speed controller is presented in Eq. (16). The speed controller, $C(\mathrm{~s})$, is designed with PI feedback control.

$$
C(s) G_{M}(s)=\frac{\Omega}{\Omega_{d}}=\frac{K_{p}\left(K_{t} / J_{\text {total }}\right)\left(s+K_{i} / K_{p}\right)}{s^{2}+\left(B_{\text {tot }} / J_{\text {total }}\right) s}
$$

(Values for $K_{t}, J_{\text {total }}$, and $B_{t o t}$ are $0.31 \mathrm{~N}-\mathrm{m} / \mathrm{A}, 79.3 \mathrm{~kg}-\mathrm{cm}^{2}$, and $1.06 \mathrm{~N}-\mathrm{cm}-\mathrm{s}$, respectively.)

The integral and proportional gains, $K_{i}$ and $K_{p}$, were determined using root loci and simulation techniques. To avoid significant speed error associated with an underdamped oscillatory speed response, all roots for Eq. (16) were designed to be on the negative real axis. From a frequency domain perspective, it was desirable to have a high ratio of integral to proportional gain. This was realized by placing the transmission zero farther along the negative real axis away from the origin. Values of 5 through 10 for the $K_{i} / K_{p}$ ratio were determined effective through root locus design and verified through simulation. Stable values of $K_{p}$ were found from 0.3 to 30. Figure 10 depicts typical continuous root loci for $K_{i} / K_{p}=5$. A non-oscillatory response can be achieved by keeping the system poles on the real axis.

Another important controller design consideration is the discrete system stability. As controller update rates range from 1 to $20 \mathrm{kHz}, 1 \mathrm{kHz}$ sampling was used to assess stability. The discrete model of the continuous system of Eq. (16), given by Eq. (17), was determined assuming a zero order hold on the input. A value 10 was assumed for $K_{i} / K_{p}$.

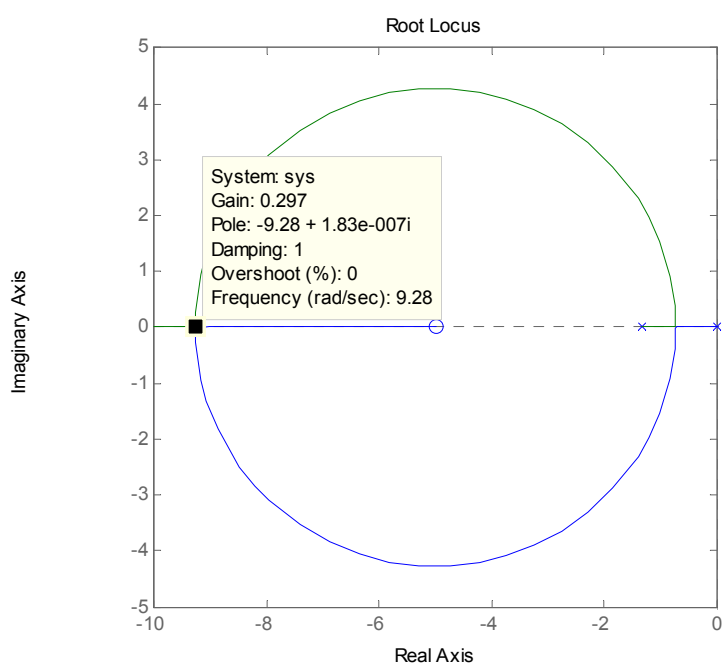

Figure 10. Continuous root loci

$$
C(z) G_{M}(z)=\frac{K_{P}(0.03924 z-0.03885)}{z^{2}-1.9987 z+0.9987}
$$

Stability was found for values of proportional gain for which a critical damped response was achieved (with values of 5 and 10 for $K_{i} / K_{p}$ ). The design constraint of a non-oscillatory response was more limiting than discrete stability. Results for acceptable values of $K_{p}$ from the discrete root locus design were found to be nearly identical to those obtained from the continuous root locus design due to the relatively fast sampling.

\section{RESULTS}

Simulation studies were performed with a Simulink ${ }^{\mathrm{TM}}$ model of the capstan speed control subsystem shown in Fig. 11. Since fiber diameter is a function of the capstan speed, the velocity error may be related directly to the fiber diameter error (Eq. (14)). A 4\% ramp increase in the line speed set point over $100 \mathrm{~s}$ was modeled and simulated to represent the preform diameter input change. Line speed error, diameter error, and motor current were adjusted for various gains modeling the $200 \mathrm{~mm}$ capstan pulley and motor 3 . The purpose of the simulation was to assess the capstan speed controller design with anticipated inputs.

Figure 12 depicts the results of four simulation cases for (a) $K_{p}=30, K_{i} / K_{p}=5$, (b) $K_{p}=3, K_{i} / K_{p}=5$, (c) $K_{p}=0.3, K_{i} / K_{p}=5$, (d) $K_{p}=3, K_{i} / K_{p}=10$. From these results the design with a 200 mm capstan pulley and motor 3 using $K_{p}=3, K_{i} / K_{p}=10$ meets the performance specifications. The settling time for speed was under $0.5 \mathrm{~s}$ while power and current limits were not exceeded. Given these favorable results, the gains of $K_{p}=3$ and $K_{i} / K_{p}=$ 10 were selected as the final design gains for the speed controller. 
The fiber diameter error is well within tolerances. However, as noted earlier, the present model does not include other processing variables and effects, such as variations in preform feed speed or furnace temperature, sensor noise, and measurement time delay. These may be examined in future work.

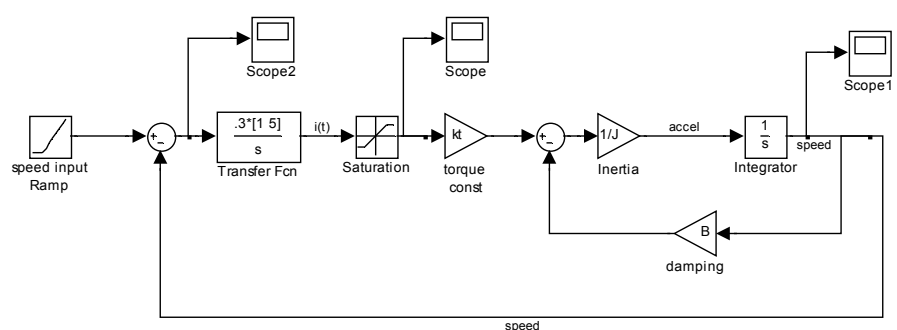

Figure 11 Simulink $^{\mathrm{TM}}$ model of speed control loop
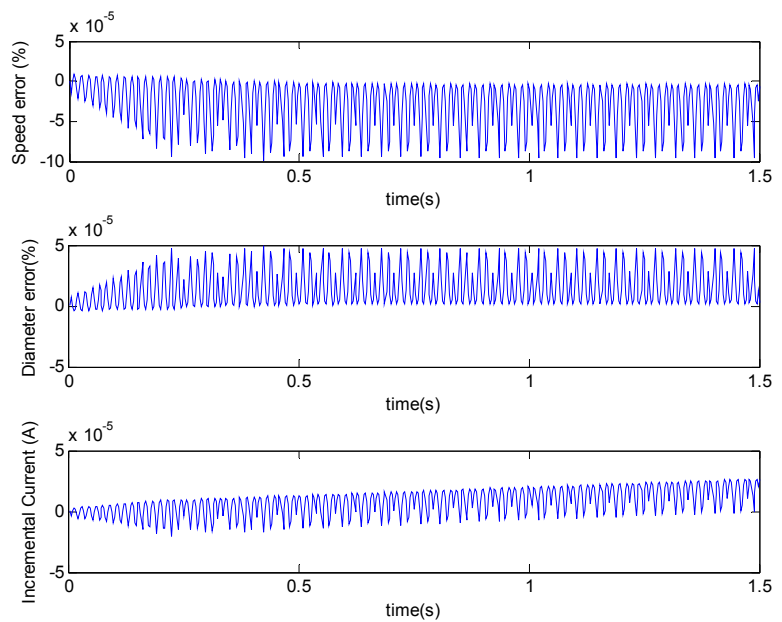

Figure 12(a). Simulation results: Line speed error and motor current for $\mathrm{K}_{\mathrm{p}}=30, \mathrm{~K}_{\mathrm{i}} / \mathrm{K}_{\mathrm{p}}=5$
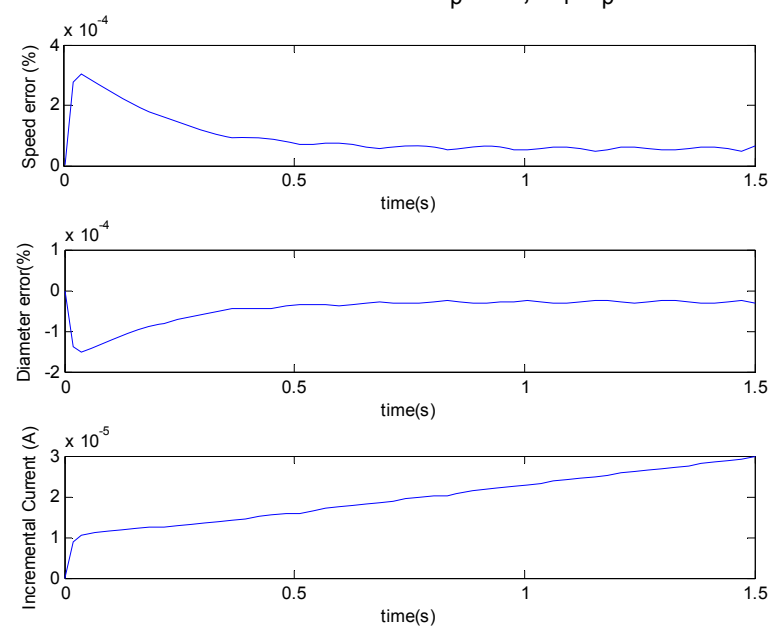

Figure 12(b). Simulation results: Line speed error and motor current for $\mathrm{K}_{\mathrm{p}}=3, \mathrm{~K}_{\mathrm{i}} / \mathrm{K}_{\mathrm{p}}=5$
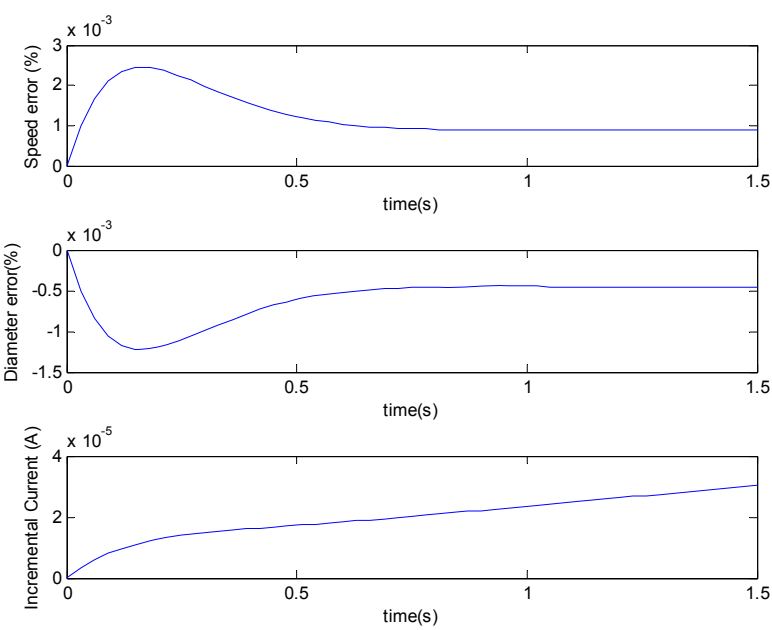

Figure 12(c). Simulation results: Line speed error and motor current for $\mathrm{K}_{\mathrm{p}}=0.3, \mathrm{~K}_{\mathrm{i}} / \mathrm{K}_{\mathrm{p}}=5$
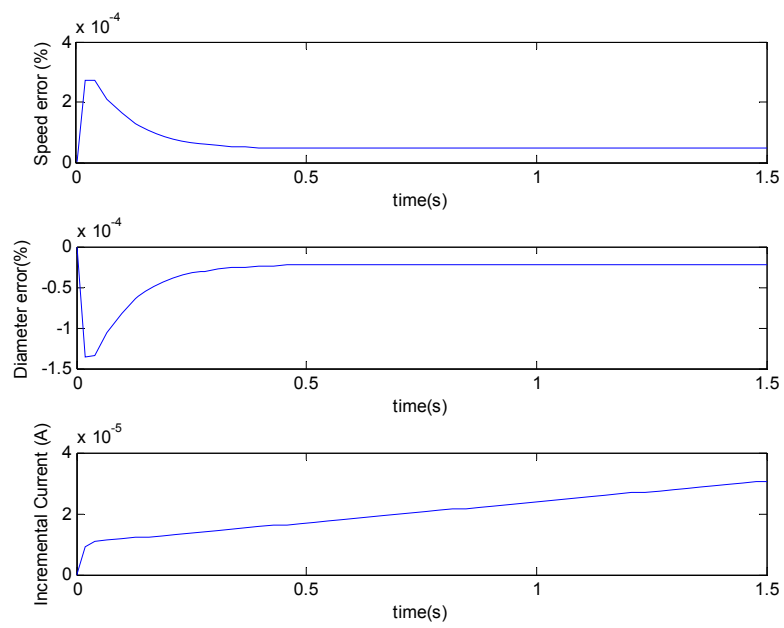

Figure 12(d). Simulation results: Line speed error and motor current for $\mathrm{K}_{\mathrm{p}}=3, \mathrm{~K}_{\mathrm{i}} / \mathrm{K}_{\mathrm{p}}=10$

\section{CLOSING}

The design of an optical fiber draw capstan pulley including the motor selection and speed control system has been conducted to improve optical fiber diameter control. Through simulation studies, a stable, exponential response was achieved, without motor current saturation, for the capstan speed in the presence of estimated process disturbances. With the design and process relationships established, an effective draw capstan system has been modeled, designed, and controlled and shown to offer performance advantages.

The design solution for the capstan system is predicated on a fundamental understanding of mechanical, electrical, and control theory to solve a real-world engineering problem. The modeling of the process and the selection of the design parameters using multi-disciplinary criteria resulted in a machine that potentially performs better than that possible by serial (i.e., sequential) design. 
Although specific hardware and software systems change, the fundamental engineering concepts underlying the successful design of a device, machine, or process remain relatively constant. This case study offers an effective approach for machine design and process control that relies on modeling, analysis, and simulation. A successful method of design with simulation was built upon (1) problem definition, (2) determination of parameters that affect control, (3) system model techniques, (4) physical and power limitations, (5) frequency domain control design, and (6) discrete control design. Due to its multi-disciplinary nature, this design case study has broad applications to practical engineering applications that include control, electrical, and mechanical systems, typical in modern manufacturing.

\section{NOMENCLATURE}

$B$ rotational damping

$d \quad$ fiber diameter

I motor current

$J \quad$ polar mass moment of inertia

$K_{i} \quad$ integral gain

$K_{p} \quad$ proportional gain

$K_{t} \quad$ motor torque constant

$M \quad$ mass

$Q \quad$ volumetric flow rate

$r \quad$ outside radius of capstan pulley

$t$ time

$\tau \quad$ thickness of capstan pulley rim

$v \quad$ belt speed $(\omega \times r)$

$\omega$ motor rotational speed

$\dot{\omega}$ motor rotational acceleration

$\Omega(s)$ Laplace transform of $\omega(t)$

\section{REFERENCES}

[1] Auslander, D. M., "What is Mechatronics?" IEEE Transactions on Mechatronics, Vol. 1, No. 1, 1996.

[2] Panaitescu, R., "Improving machine designs with mechatronics," Design News, September 25, 2006.

[3] Jenkins, H.E. and Nagurka, M.L., "Teaching a SeniorLevel Mechatronics Course in Mechanical Engineering," 2004 Japan-USA Symposium on Flexible Automation, Denver, CO, paper UL-047, 2004, pp.1-7.

[4] Refi, J., Fiber Optic Cable: A Light Guide, ABC Teletraining, Geneva, IL, 1991.

[5] Li, T. (Ed.), Optical Fiber Communications, Vol. 1, Academic Press, Inc., Orlando, FL, 1985.

[6] MacChesney, J.B., O'Connor, P.B., and Presby, H. M., "New Technique for the Preparation of Low-Loss and Graded Index Optical Fibers," Proc. IEEE, Vol. 62, No. 9, 1974, pp. 1282-3.

[7] URL:http://www.berkeleyprocess.com

[8] Ljung, L., System Identification: Theory for the User, Prentice Hall, Englewood Cliffs, NJ, 1987.

[9] Shearer, J.L., Kulakowski, B.T. and Gardner, J.F., Dynamic Modeling and Control of Engineering Systems, Second ed., Prentice-Hall, 1997, p.219. 